\title{
Alzheimer Demenz: Bedeutung der perineuronalen extrazellulären Matrix (PECM)
}

\author{
Hartmut Heine \\ Professor für Anatomie, Histologie und Embryologie, ECM-Forschung, DE-Neuhausen
}

$\mathrm{V}$ or 100 Jahren (1906) sezierte AloIs AlzHEIMER (Klinik für Psychiatrie und Psychotherapie, München) das Gehirn seiner ehemaligen Patientin Auguste D. und beschreibt „eine eigenartige Erkrankung der Hirnrinde“ zunächst ohne jedes Echo [52]. 1910 führte der damalige Direktor der Klinik Prof. Dr. Kraepelin die Bezeichnung Alzheimer Krankheit im Lehrbuch der Psychiatrie ein [52].

„Oh wie schön wäre es doch, wenn ich den Schädel nicht immer öffnen müsste“ - dieser Wunsch von Alors AlzheIMER (1864-1915) zur Diagnostik der nach ihm benannten Demenz ist mittlerweile Wirklichkeit [35].

$\mathrm{Zu}$ verschiedenen Hirnleistungstests z.B. der Uhr-Zeichen-Test, MiniMental-Status-Test [Rem Tect. $\left.{ }^{\circledR}\right]$, Protein-Biomarkern im Liquor und Blut [52] kommen funktionelle bildgebende Verfahren wie SPECT (Single Photon Emisssion Computertomography), PET (Positronenemissionscomputertomographie), FDG (18 Fluor-deoxy-glukose) und vor allem die fMRT (funktionelle Magnet-Resonanz-Computertomographie) [35].

Zusätzlich können Bestandteile der Alzheimer-Plaques sowie neurofibrillärer Bruchstücke in Spuren im Liquor nachgewiesen werden [52]. In der Erforschung der Alzheimer Demenz (AD) steht die erbliche Komponente im Vordergrund. Sie macht jedoch nur etwa $5 \%$ der AD-Fälle aus $[17,20]$. Die dabei gewonnenen Erkenntnisse werden ohne Diskussion auf die nichterbliche AD übertragen [24].

Nahezu jede Krankheit entsteht aus dem Zusammenwirken von genetischen und Umweltfaktoren. Dominante Erbkrankheiten stellen dagegen nur ca. 3\% aller Erkrankungen dar [48].
Alzheimer Demenz (AD) ist die verbreitetste neurodegenerative Erkrankung im Alter. Die Erforschung der AD ist nahezu vollständig auf den gestörten Stoffwechsel von Neuronen gerichtet. Das die Neuronen umgebende Milieu (extrazelluläre Matrix ECM) wird dabei praktisch nicht beachtet. In diesem Beitrag wird eine neue Theorie vorgestellt, die der Autor aus seiner umfangreichen Forschungstätigkeit entwickelt hat. Diese Theorie wird gestüzt und ergänzt durch eine Analyse der Literatur (1981-2006) aus den gängigen elektronischen Datenbanken sowie aus Standardwerken und Monographien. Im Mittelpunkt der Erforschung steht heute die erbliche AD, die aber lediglich $5 \%$ der Fälle ausmacht. Die bei der erblichen $A D$ erzielten Forschungsergebnisse werden diskussionslos auf die 95\% der nichterblichen $A D$ übertragen. Nach der Theorie des Autors steht bei nichterblicher AD der Astrozyt - und nicht das Neuron - im Zentrum des Geschehens. Dieser Zelltyp bildet das auf die perineuronale ECM beschränkte Proteoglykan Appican, dessen Proteinrückgrat das für die AD-Entwicklung entscheidende Amyloid-Precursor-Protein (APP) darstellt. Diese Sichtweise erlaubt den Rückschluss, dass pathogenetisch hauptsächlich die Appican-bildenden Astrozyten für die Entwicklung der nichterblichen AD verantwortlich sind. Demzufolge werden Neurone über Astrozyten als 'Ammen' mit den für den neuronalen Stoffwechsel wichtigsten Enzymen und Metaboliten versorgt. Altern als subklinischer proinflammatorischer Prozess stört diese Beziehungen. Zuerst sind davon die schwach myelinisierten Assoziationsbahnen im Gehirn betroffen. Gleichzeitig werden Proteasen aktiviert, wodurch aus Appican zunehmend APP freigesetzt wird. Damit wird die Plaquebildung bei AD forciert. Diese Prozesse werden von einer neurotoxischen Zunahme an Ammoniak im Gehirn begleitet. Als therapeutische Option wäre es daher möglicherweise sinnvoll, zunächst überschüssigen Ammoniak abzufangen, um den Verlauf der AD therapeutisch zu beeinflussen.

Schlüsselwörter: Alzheimer, Astrozyten, Appican, Assoziationsbahnen, Ammoniak

\section{Alzheimer Disease: Role of the Perineuronal Extracellular Matrix (PECM)}

Alzheimer's disease (AD) is the most common neurodegenerative disease in the elderly. Scientific research centers on neurons whereas their extracellular milieu (extracellular matrix ECM) is nearly neglected. This essay presents new aspects resulting from the author's own extensive scientific research supported by an analysis of the literature from electronic data bases (1981-2006), standard books and monographs. Current research focuses on the inherited form of AD which accounts for only $5 \%$ of the disease. The findings are transmitted, without being discussed, to the $95 \%$ of the non-inherited form of $A D$. In the author's opinion, not the neuron but the astrocyte is the functional centre point in non-inherited AD. Astrocytes are capable of synthesizing the proteoglycan Appican (APP) which is unique for the perineuronal ECM. The protein backbone of APP represents the amyloid precursor protein which is decisive for the pathogenesis of $A D$ plaques. From this, the conclusion could be drawn that astrocytes are playing the central part in non-inherited AD. Moreover, astrocytes behave as 'nurses' for neurons by supplying them with important enzymes and metabolites. Aging can be interpretated as a subclinical proinflammatory process that, in the course of time, disturbs the relations between the named cells. The first to be attacked are the neurons of the slightly myelinated association tracks in the brain. Simultaneously, proteases of the perineuronal ECM are activated, cleaving APP and accelerating the formation of $A D$ plaques. These processes are accompanied by a neurotoxic augmentation of ammonia in the brain. From a therapeutic point of view it might be useful to first neutralize excessive ammonia in order to therapeutically influence the progess of AD.

Keywords: Alzheimer's disease, astrocytes, appican, association tracks, ammonia 
Ziel dieser Arbeit ist es zu zeigen, dass in der Pathogenese der $\mathrm{AD}$ die Rolle der extrazellulären Matrix im Gehirn (perineuronale ECM, PECM) stärker beachtet werden sollte. Diese ist allen Nervenzellen und ihren Fortsätzen als Molekularsieb vorgeschaltet und somit der Knotenpunkt für alle Umwelteinflüsse [24].

\section{Alter und Alzheimer Demenz}

Hauptrisikofaktor für die Entwicklung der $\mathrm{AD}$ ist Alter. $\mathrm{AD}$ ist die verbreitetste neurodegenerative Erkrankung im Alter. In Deutschland gibt es zur Zeit etwa 1 Million Personen mit AD [18]. Nach Hesch [25] ist in Deutschland mit einer Inzidenz von jährlich 50.000 Neuerkrankungen zu rechnen. In den USA waren im Jahr 2000 etwa 4,5 Millionen erkrankt [11,17]. Die Behandlungskosten wurden für 1996 auf 83,9 Milliarden Dollar geschätzt [11] (für Deutschland muss dann mit etwa 3,5 Milliarden gerechnet werden). Der Prozentsatz an Personen mit $\mathrm{AD}$ wächst ab dem 60 Lebensjahr (mit ca. 1\% Erkrankter) alle 5 Jahre um den Faktor 2 an; d. h., dass ca. 30\% der 85-Jährigen betroffen sind [11,38]. Ohne Fortschritte in der Therapie werden sich die AD-Fälle in den USA bis zum Jahr 2050 auf 13,2 Millionen [11], umgerechnet bei uns auf über 3 Millionen erhöhen. Da die Lebenserwartung bei Frauen seit 1950 gegenüber den Männern stärker angestiegen ist, ist der grösste Teil alter Menschen weiblich. In hohem Alter (> 80 Jahre) ist die Sterblichkeitsrate bei Männern gegenüber Frauen dreifach höher [37]. Daher ist auch der Anteil von Frauen mit AD höher [25]. Dazu kommt, dass nach der Menopause die organprotektive Wirkung der Östrogene abnimmt und das Risiko an Alzheimer zu erkranken erheblich zunimmt [25].

\section{Entwicklung}

\section{der Alzheimer-Demenz}

Abgesehen von den ca. 3\% dominant erblichen Krankheiten, stellt die überwiegende Mehrzahl von Krankheiten Fehler in sich selbstorganisierenden organischen Netzwerken dar, in die alle Parameter des aktuellen Lebens eingehen [41]. Daraus resultiert eine individuelle Kritizität als Grenze von Gesundheit zu Krankheit [41]. Alter stellt dabei den führenden Risikofaktor im Absinken dieser Kritizität dar mit Auftreten von Altersdiabetes, HerzKreislauf-Erkrankungen, Hypertonie, Hypercholesterinämie, Adipositas, Osteoporose, grauem und grünem Star, Demenzen, Tumoren, Schmerzen verschiedenster Genese usw. [17,32]. Dazu tragen auch exogene Faktoren bei, wie falsche, zu hochkalorische Ernährung, Umwelteinflüsse wie organische Schadstoffe, Schwermetallionen, Stress durch Lärm, ungeeignete Wohnung u. a. m. [24,37]. Die genannten pathophysiologischen Faktoren schaffen eine proinflammatorische Situation mit Bildung reaktiver Sauerstoffspezies (ROS) und Aktivierung entzündungsfördernder Zytokine. Im Mittelpunkt steht der Tumornekrosefaktor-alpha $(\mathrm{TNF}-\alpha)$. Dieses Zytokin bedingt Insulinresistenz, Aktivierung von Osteoklasten, Hypertonie mit allen Folgen für das Herz-Kreislauf-System und die Störung der mittels Leptin aus Fettzellen über die Hypophyse gesteuerten hormonellen Nahrungsverwertung $[15,20]$. Altern kann daher als proinflammatorischer Prozess mit sehr individueller Ausprägung gesehen werden. Dies ist offensichtlich eine Voraussetzung für die Entwicklung der AD [22]. Dazu kommt, dass Menschen mit zwei Allelen des ApoE4-Gens stets ein erhöhtes Erkrankungsrisiko haben [11,32,52].

Das komplexeste Netzwerk in unserem Körper ist das Nervensystem und besonders die Gehirnrinde (Neocortex). Die abgestimmte Verarbeitung von Aussen- und Inweltreizen mit emotionaler Abstimmung wird durch das limbische System geregelt. Dieser Gehirnbereich verbindet und koordiniert den stammesgeschichtlich alten Allocortex, u. a. Riechhirn, Hippocampus, Mandelkern, über das Cingulum mit dem Neocortex [57] (Abb. 1). Die gegenseitigen Verbindungen erfolgen über die stammesgeschichtlich jungen Assoziationsbahnen (Abb. 1). Diese langen Bahnen sind aufgrund ihres späten Auftretens in der Stammes- und Entwicklungsgeschichte auch nur schwach myelini- siert und daher besonders vulnerabel [6]. Sie koordinieren u. a. die Gebiete für Lern- und Gedächtnisfunktionen sowie für soziales Erfolgs- bzw. Verlustverhalten [6]. Die wichtigsten Neurotransmitter entlang dieser Neuronenketten sind die exzitatorischen Transmitter Glutamat, Acetylcholin und Noradrenalin [57].

„Die Nachteile der flächenmässig gewaltigen Entwicklung einfach gebauter, schwach myelinisierter Assoziationsgebiete höherer Ordnung liegen in der partiellen Unausgereiftheit dieser zum Teil erst in späten Phasen der Primatenentwicklung entstandenen Felder“ [57]. In der Entwicklung der $\mathrm{AD}$ sind daher zuerst die markarmen Assoziationsbahnen betroffen [6] (Abb. 1). Es wäre zu diskutieren, ob nicht bereits im frühen Kindesalter durch Deprivation, d. h. jede Form von Stress und das dadurch bedingte neuronale Sauerstoffdefizit, die ohnehin geringe Myelinisierung der Assoziationsbahnen gestört wird, was zeitlebens eine individuelle Vulnerabilität dieser Bahnen verursachen würde [6, 7]. Bis sich jedoch ein summierendes Funktionsdefizit über unvermeidbaren „Lebensstress“ im Sinne einer sporadischen AD entwickelt, können mehr als 50 Jahre vergehen [6]. Da die Myelinisierung der Nervenbahnen bis zum 7. Lebensjahr abgeschlossen ist [7], kann darüber spekuliert werden, ob nicht ein grosser Teil von $\mathrm{AD}$ bereits im frühen Kindesalter angelegt wird und durch kindgerechte Erziehung, z. B. liebevolle Zuwendung, Schulung der Aufmerksamkeit und initiatives Verhalten durch Spielen sowie Entwicklung der Sensibilität und Motorik (z. B. durch Musizieren) vermieden werden könnte [6,24]. Dazu liegen bislang keine Studien vor.

\section{Die perineuronale extra- zelluläre Matrix (PECM)}

$\mathrm{AD}$ ist nicht von der fortschreitenden alternsbedingten Sklerose der Gehirnarterien und der physiologischen alterungsbedingten Gefässrückbildung zu trennen [53] (Abb. 2).

Durch die dabei auftretenden Durchblutungsstörungen entstehen Versor- 


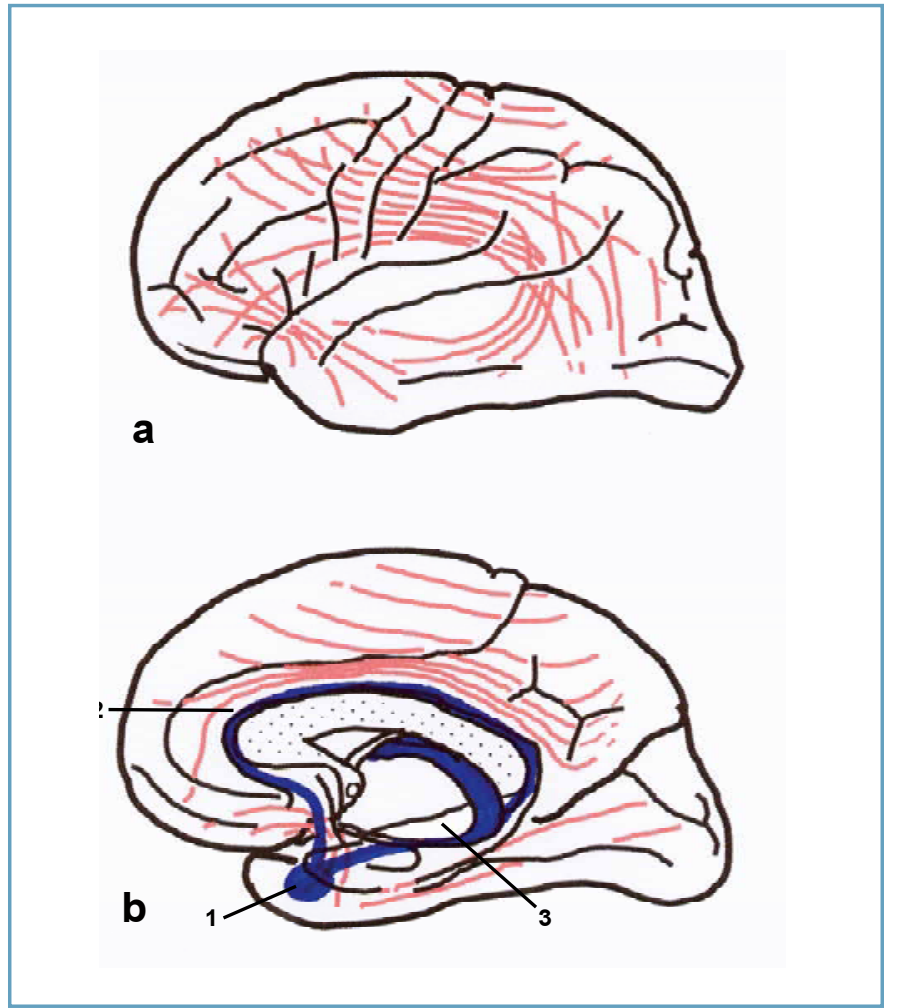

Abb. 1. Lange Assoziationsbahnen projiziert auf die laterale (a) und mediale Hirnfläche (b). In b sind wichtige Zentren des limbischen Systems (blau) dargestellt. 1 Mandelkern (Amygdala), 2 Cingulum, 3 Hippocampus.

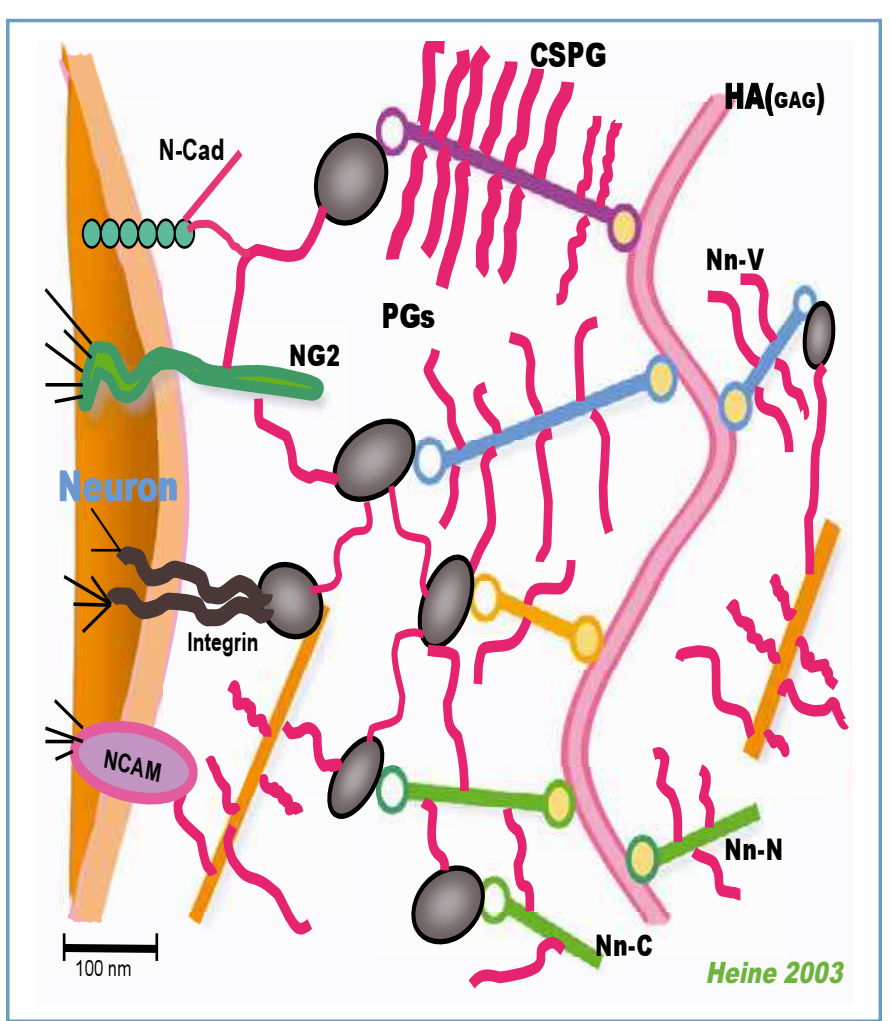

Abb. 2. Perineuronale extrazelluläre Matrix. Die PECM wird von Proteoglykanen (PGs), Glykosaminoglykanen (GAG; Hyaluronsäure HA), Matrix-PGs (u. a. Appican) und Strukturglykoproteinen (schwarze Ellipsen) gebildet. Der neuronale Zellzuckeroberflächenfilm enthält Heparansulfat PGs (NG2), Integrine und Zelladhäsionsmoleküle (N-Cad, N-Cadherin, NCAM neuronales Zelladhäsionsmolekül). Diese Moleküle stehen mit dem Zytoskelett (kurze schwarze Linien) in Verbindung. CSPG Chondroitinsulfatprotein, Nn-C, Nn-N, NnV Spaltprodukte von PGs. gungsengpässe, von denen besonders die Assoziationsbahnen betroffen sind [22]. Dazu kommt, dass im Bereich dieser Bahnen mit fortschreitendem Alter der gesamte Gefässbaum eine Reduktion erfährt [9].

Zwischen Kapillaren und den zu versorgenden Zellen ist, bis auf die zirkumventrikulären Organe, eine BlutHirn-Schranke entwickelt, die das interne Milieu des Nervengewebes stabilisieren hilft. Die Schranke besteht aus Kapillarendothelzellen, unterlagernder Basalmembran (ca. $60 \mathrm{~nm}$ dick) und ist gegen das Nervengewebe von einer lückenlosen Schicht schuhartig verbreiterter Astrozytenfortsätze bedeckt [57]. Die Endothelzellen überwachen enzymatisch den Stoffdurchtritt vor allem von Glukose, wogegen Sauerstoff frei übertreten kann. Die Metaboliten werden von den Astrozytenfortsätzen aufgenommen und Kataboliten in den venösen Kapillarschen- kel abgegeben [32]. Die Astrozyten nehmen u. a. an der Steuerung der Ionenkonzentration im Extrazellulärraum teil, der in der Hirnrinde immerhin 20\% des Gesamtvolumens einnimmt. Daher werden Astrozyten für die Aufrechterhaltung eines konstanten Ionenmilieus im Gehirngewebe verantwortlich gemacht [32]. Die Oligodendrozyten stellen eine weitere Gliazellform dar, die die Myelinisierung der Nervenfasern besorgen. Mikrogliazellen, die Phagozyten im Zentralnervensystem (ZNS), entstehen aus Blutmonozyten, die während der Embryonalzeit in das ZNS einwandern [32].

Für die Entwicklung der $\mathrm{AD}$ ist die mit zunehmendem Alter sich verbreiternde Basalmembran (beim 85-Jährigen um $50 \%$ gegenüber Jugendlichen) als gestörter Diffusionsfilter von Bedeutung [22,55]. Der sich anschliessende PECM-gefüllte Extrazellulärraum kann einige Nanometer bis wenige Mi- krometer breit sein [22]. Die den Raum strukturierenden Makromoleküle lassen sich unterscheiden in Proteoglykane (PGs) mit einem ca. 300 nm langen Proteinrückgrat, an das Polysaccharidseitenketten gebunden sind und Glykosaminoglykane (GAGs) als nicht sulfatierte, gestreckte, bis $\mathrm{zu} 1 \mu \mathrm{m}$ lange, polymere Zuckerketten ohne Proteinrückgrat. Im Gehirn tritt als GAG nur Hyaluronsäure auf (Abb. 2) [34, Übersicht bei 24].

Die PG/GAGs werden durch Vernetzungsglykoproteine (Fibronektin, Tenascin, Thrombospondin u.a.m.) untereinander verbunden. Unter den PGs haben das zellmembranständige Heparansulfat-PG (HSPG) und das hyaluronsäuregebundene Chondroitinsulfat-PG (ChSPG) mit den dazwischen vermittelnden Matrix-PGs besondere Bedeutung [16,22] (Abb. 2). Aufgrund ihrer Wasserbindungs- und Ionenaustauscherfähigkeiten sind die PG/GAGs 
Garanten für Isoionie, Isoosmie und Isotonie in der PECM. Darüber hinaus ist die PECM ein dynamischer Speicher für Zytokine, Wachstumsfaktoren, Enzyme (u. a. Proteasen, Proteaseninhibitoren), Komplement- und Gerinnungsfaktoren, Eicosanoide, Transportlipoproteine, wie das Apolipoprotein E, und Adhäsionsfaktoren [15,32]). Die momentane Situation im ZNS spiegelt sich daher in der Zusammensetzung der PECM wieder [24].

Im ZNS werden die PG/GAGs von Astrozyten und in geringerem Umfang von den Mikrogliazellen gebildet [32, 34]. Eine Besonderheit stellt die Synthese des Matrix PGs Appican dar, das praktisch nur von Astrozyten (ca. 1\% von Neuronen und Mikrogliazellen) gebildet wird [32,39] (Abb. 3).

Auf jede Veränderung in der PECM reagieren die Astrozyten mit einer situationsgerechten Synthese von PECM. Sie können jedoch nicht die Strukturglykoproteine Kollagen und Elastin bilden [22,34]. Diese werden durch Appican ersetzt, das als Proteinrückgrat das für die Entwicklung der sporadischen $\mathrm{AD}$ entscheidend wichtige Amyloidpräkursor- (Vorläufer-) Protein (APP) enthält [Übersicht bei 24].

Von besonderer Bedeutung für die Molekularsiebwirkung und Informationsleitung in der PECM sind die ChSPGs und HSPGs. Die ChSPGs hemmen das Neuritenwachstum und halten dadurch die Nervenzellmembranen auf Distanz [14,29,45]. Die zellmembranständigen HSPGs, wie das Syndecan, helfen auf der neuronalen Zellmembran Hormone, Wachstumsfaktoren und Zytokine so zu akkumulieren, dass sie in raum-zeitlich geeigneter Weise ihren Rezeptoren zur Verfügung gestellt werden können. Da sie an ihre Heparansulfatketten $\mathrm{Ca}^{2+-I o n e n ~ b i n-~}$ den und damit vorrätig halten können, sind sie für die regelhafte Depolarisation und synaptische Aktivität neuronaler Membranen von grosser Bedeutung [19,29]. Die PECM ist zwischen Neuronen und Gliazellen ausgespannt, wodurch diese, nach Art gekoppelter Federn, unter eine die Zellfunktionen fördernde Vorspannung der PECM geraten $[8,22,28]$. Haftstellen zwischen Nervenzellen und PECM sind membranständige PGs, Adhäsionsmoleküle, Gly- kosphingolipide und, von besonderer Effektivität, die Integrine der Nervenzellmembran [36,40,50] (Abb. 2).

Die genannten Moleküle stehen mit dem Zytoskelett in Verbindung. Dadurch können praktisch alle Zellreaktionen über die ECPM beeinflusst werden [22] (Abb. 2). Allerdings scheinen nur die Integrine in der Lage zu sein, Signale auch aus dem Zellinneren an die Rezeptoren und von dort an die PECM weitergeben zu können [16]. Generell sind Integrine in der Lage, solche PECM-Komponenten zu binden, die ein sog. RGD-Motiv (Arginin-Glycin-Asparagin) enthalten. Dazu gehören das Proteinrückgrat der PGs und die Vernetzungsglykoproteine. Die PECM erfährt dadurch eine selbstorganisierende Stabilität („Tensegrität“), die als Viskoelastizität schockabsorbierend wirkt und alle Reaktanten in der PECM in eine funktionsgerechte molekulare Konformation sowie Lage zueinander bringt [22,28,47]. Diese Grundspannung steuert auch die Genexpression der Nervenzellen [8, 28] sowie die matrixvermittelte Chemotaxis und Haptotaxis von Glia- und Mikrogliazellen [40,47].

Dies ist von besonderer Bedeutung für die Assoziationsbahnen, die zeitlich genau abgestimmt arbeitende Neuronenketten darstellen (synfire chains) [27]. Die synfire chains zeichnen sich durch synchrone Summation exzitatorischer postsynaptischer Potentiale (EPSPs) aus, wodurch die Erregungen in der Kette eine präzise Zeitabstimmung erfahren. Erst durch diese raumzeitlichen Erregungsmuster können komplexe Muster im Cortex aufgebaut werden, die die Grundlage höherer geistiger Aktivitäten sind [27].

Die amyloide Plaquebildung bei AD trifft zuerst die gering myelinisierten und daher leicht vulnerablen synfire chains der Assoziationsbahnen. Es ist daher nicht erstaunlich, dass bei Entwicklung der $\mathrm{AD}$ typischerweise als erster Hinweis Unsicherheiten im Ablesen oder Aufzeichnen eines Uhrzifferblatts mit einer bestimmten Zeigerstellung auftreten (Abb. 3). Dies ist gleichzeitig eine Bestätigung des Konzeptes der synfire chains.

\section{Die Bedeutung von Appican für die Entwicklung der Alzheimer-Demenz}

Das im Wesentlichen von Astrozyten gebildete Appican stellt ein PG mit einem Proteinrückgrat aus 695 bis 770 Aminosäuren dar, das an den Serinen 637 und 660 je eine polymere Chondroitinsulfatseitenkette trägt. Sie verhindern die Selbstaggregation des Moleküls [29,32]. Diese Serinbindungen sind ausserordentlich empfindlich gegen die Serinprotease Plasmin, wie sie bereits proinflammatorisch aus PECMgebundenem Plasminogen generiert werden kann [15,32]. Die dabei abgespaltenen kurzen Bruchstücke des Proteinrückgrats von Appican bilden nach der hier vertretenen Meinung im Wesentlichen die amyloidogenen $A \beta$ Proteine als „Kristallisationskeime“ der Alzheimer-Plaque [22,32] (Abb. 3). Daraus ergibt sich eine bisher nicht beachtete, aber sehr wesentliche Unterscheidung von nichterblicher und erblicher AD [22]: Bei der nichterblichen $\mathrm{AD}$, die ca. 95\% der Fälle ausmacht, steht der Astrozyt und nicht das Neuron im Mittelpunkt (Abb. 4).

Bei erblichem Alzheimer überwiegt dagegen die $A \beta$-Produktion durch Neurone. Dabei wird das Amyloidvorläuferprotein (APP) bereits bei Ausschleusung intramembranös durch gammaSekretasen (Presenilin) in die plaquebildenden $\mathrm{A} \beta$-Proteine gespalten $[38,39,51,56]$.

\section{Ammenfunktion der Astrozyten}

Die Bereitstellung wichtiger Enzyme des Krebs-Zyklus (u.a. $\alpha$-Ketoglutaratdehydrogenase) sowie Substrate für den Energiestoffwechsel der Neurone (Glutamin, Glutamat, Glutamatsynthetase) erfolgt über die Astrozyten (Abb. 5). Ihre Ammenfunktion in Form eines Shuttle-Mechanismus weist einen biphasischen Rhythmus auf zwischen oxidativer und glykolytischer Bereitstellung von Glukose und Milchsäure [3,4,30]. Die Energie (Elektronen) zur Generierung dieser Metaboliten wird von dem Redoxpaar NAD+:NADH bereitgestellt [30]. Dafür spricht die 


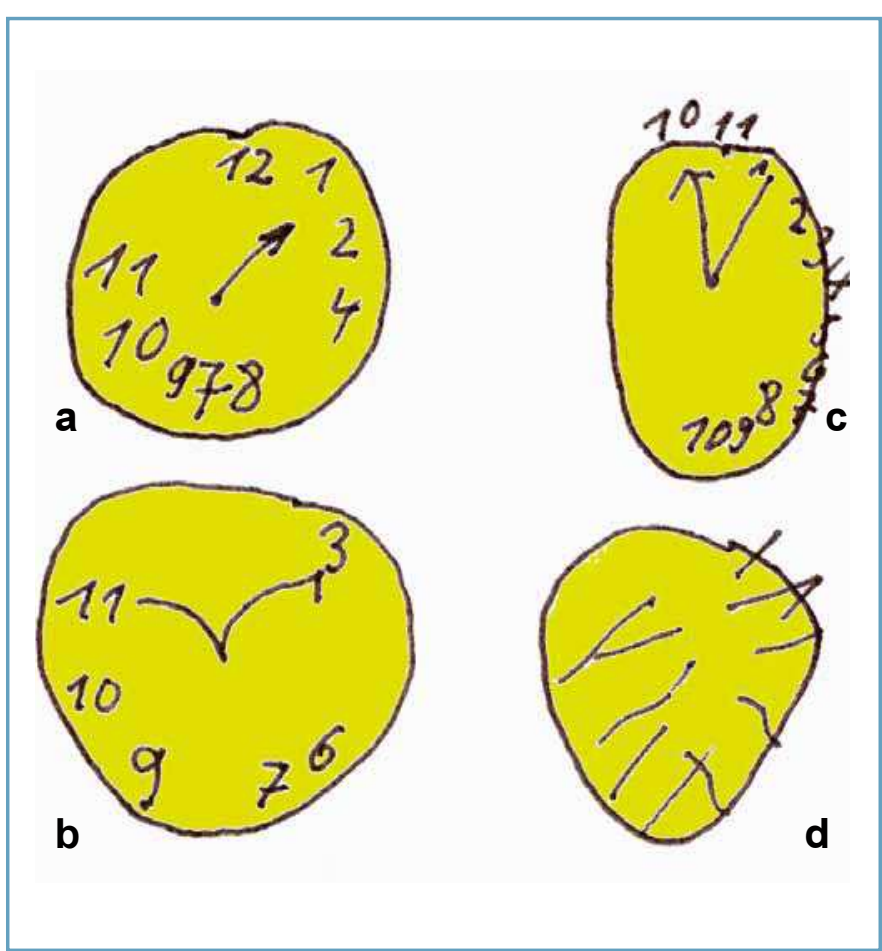

Abb. 3. a-d Zunehmend gestörtes Uhrenzeichnen bei Alzheimer-Patienten (nach [35]).

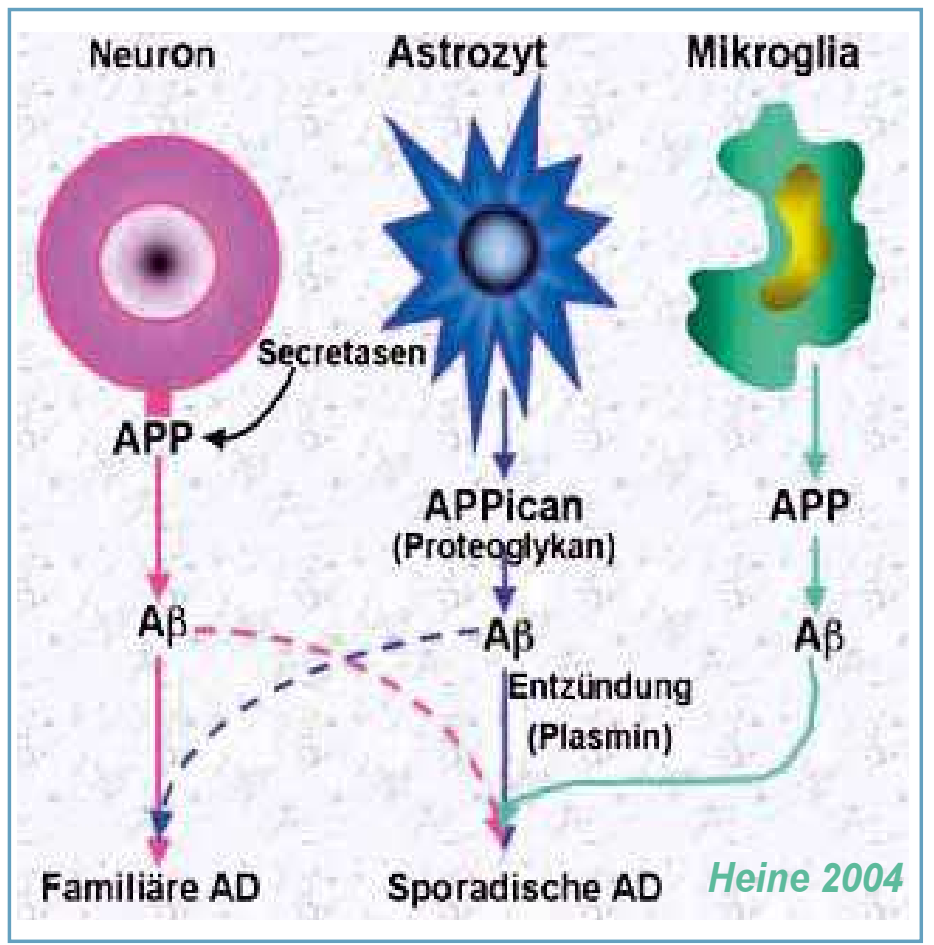

Abb. 4. Bedeutung der Astrozyten für die nichterbliche Alzheimer Demenz. Das überwiegend nur von Astrozyten gebildete Proteoglykan Appican wird unter den proinflammatorischen Bedingungen der AD extrazellulär proteolytisch (Plasmin) in das Amyloidpräkursorprotein (APP) und weiter in amyloidogene A, Proteine gespalten. Der von Mikrogliazellen gebildete geringe APP-Anteil wird extrazellulär proteolytisch gespalten. Die gebrochenen Pfeile weisen auf einen kleinen wechselseitigen zellulären Beitrag von $A \beta$ (man beachte den wichtigen Unterschied in der A $\beta$-Bildung bei nichterblicher und erblicher AD).
Periodik von etwa 10 Sekunden, da diese auch für die Bereitstellung von NADH (oder NADPH) in Neutrophilen gemessen wurde [31]. Auch das für den Membranstoffwechsel, vor allem den von Synapsen, unerlässliche Cholesterin wird von den Astrozyten an die Neurone geliefert [3,19]. Das Cholesterin wird zum Transport an Apolipoprotein E (Apo E) gebunden [19]. Isoformen des ApoE, wie das Apo E4, transportieren allerdings bevorzugt schädliche A $\beta$-Moleküle und fördern damit die AD-Entwicklung [15].

Die Ammenfunktion der Astrozyten hat auch eine Schlüsselfunktion im Umsatz der wichtigsten exzitatorischen und inhibitorischen Neurotransmitter, dem Glutamat und der gammaAminobuttersäure (GABA). Zur Glutamatbildung werden von den Astrozyten $\alpha$-Ketoglutaratdehydrogenase und Glutaminsynthetase zu den Neuronen geleitet und überschüssiges Glutamat aus dem Synapsenspalt zu den Astro- zyten zurücktransferiert [19]. Glutamat ist auch die Ausgangssubstanz für GABA. Überschüssig freigesetzte Neurotransmitter sowie „abgenutzte“ Rezeptoren der postsynaptischen Membran können über die angrenzende Astrozytenmembran aufgenommen und $\mathrm{zu}$ zelleigenen sowie wieder ausschleusbaren Produkten weiterverarbeitet werden [4,19]. Dabei kann u. a. Glutamat $\mathrm{zu}$ Glukosamin umgebaut werden, das wiederum Grundbaustein für die PG/GAG-Synthese ist [32].

Prä- und postsynaptische Membranen werden für den Shuttle-Prozess eng von Astrozytenfortsätzen umgeben (Abb. 5). Der Spaltraum zu den synaptischen Membranen ist nicht grösser als ca. 20 bis $50 \mathrm{~nm}$. Der Raum wird von den Membranoberflächenmolekülen (Glykokalyx) der sich gegenüberliegenden Zellmembranen überbrückt (Abb. 5). Dabei werden geordnete Kompartimente gebildet, die als Mikrodomänen bezeichnet wer- den [19]. Bei deren Gestaltung spielen membranständige HSPGs und Adhäsionsmoleküle (N-CAMs und N-Cadherine) eine entscheidende Rolle (vgl. Abb. 1) [2, 5, 6, 13]. Die gegenüberliegenden negativ geladenen HSPGs bilden untereinander Brücken, die den Stofftransport von Proteinen und Ionen zum Neuron regeln, während die Adhäsionsmoleküle durch homophile Bindungen (gemeinsame Hydrathülle) die Membranen koppeln und auf den jeweils funktionell geeigneten Abstand einstellen [13,22]. Die N-Cadherine sind die Nervenzellmembran einfach durchsetzende Glykoproteine, die eine Schlüsselrolle bei $\mathrm{Ca}^{2+-a b h a ̈ n-~}$ giger Zellhaftung spielen [33], wobei das Kalzium in den HSPGs vorrätig gehalten werden kann (s. o.). Darüber hinaus werden N-Cadherine mit Lernen und Erinnerung in Zusammenhang gebracht [33]. Über die Mikrodomänen wird daher das kinetische Profil der Synapsen und damit die zeit- 
liche Auflösung des Informationstransfers gesteuert. Werden, wie bei AD, die perisynaptischen Mikrodomänen destruiert, gehen auch die betroffenen Synapsen unter [13,19] (Abb. 4).

\section{Alzheimer-Demenz - eine auf das Gehirn begrenzte unspezifische Entzündung}

Untersuchungen der ECM am gealterten Gelenkknorpel haben gezeigt, dass es im Alter bei den PG/GAGs zu einer Verkürzung des Proteinrückgrats sowie der Zuckerseitenketten kommt. Auch die HA wird kürzer [49]. Da die ECM der Peripherie qualitativ die gleichen PG/GAGs - bis auf Appican - wie das ZNS aufweist [16], sind die Befunde an der ECM des Knorpels prinzipiell auf die PECM übertragbar [22,39]. Während die Verkürzung der Proteinketten auf eine zunehmende alternsbedingte Aktivierung proteolytischer Enzyme zurückzuführen ist [22], ist bei Polysaccharidketten dagegen eine Abnahme der Glutaminsynthetase im Alter zu beobachten [15, 32]. Durch Mangel an diesem Enzym steht der Grundbaustein der Zuckerpolymeren der PG/GAGs, das Glukosamin, nicht mehr ausreichend zur Verfügung [15]. Im ZNS tritt Glutaminsynthetase nur in Astrozyten auf [32]. Zur Bildung von Glutamin, Glutamat und Asparagin verbrauchen Astrozyten $98 \%$ des von peripher ins ZNS gelangenden und des vor Ort gebildeten Ammoniaks [32]. Bei Entwicklung der $\mathrm{AD}$ wird wegen Störung der Ammenfunktion der Astrozyten die Glutaminsynthetase zusätzlich stark verringert (um ca. 40\%) [32]. Endeffekt ist ein erheblicher Überschuss des unverbrauchten neurotoxischen Ammoniaks [32]. Die exzitatorischen glutamatergen Synapsen der leicht vulnerablen Assoziationsbahnen sind daher auch als erste betroffen. Dabei machen sich Glutamatrezeptoren der postsynaptischen Membran „selbständig“ und initiieren irreguläre Erregungen [22,24, 43]. Dadurch wird ein synchrones synaptisches Feuern in den betroffenen Assoziationsketten zunehmend unmöglich. Bei den acetylcholinergen Synapsen ist es ähnlich, weil die Neu-

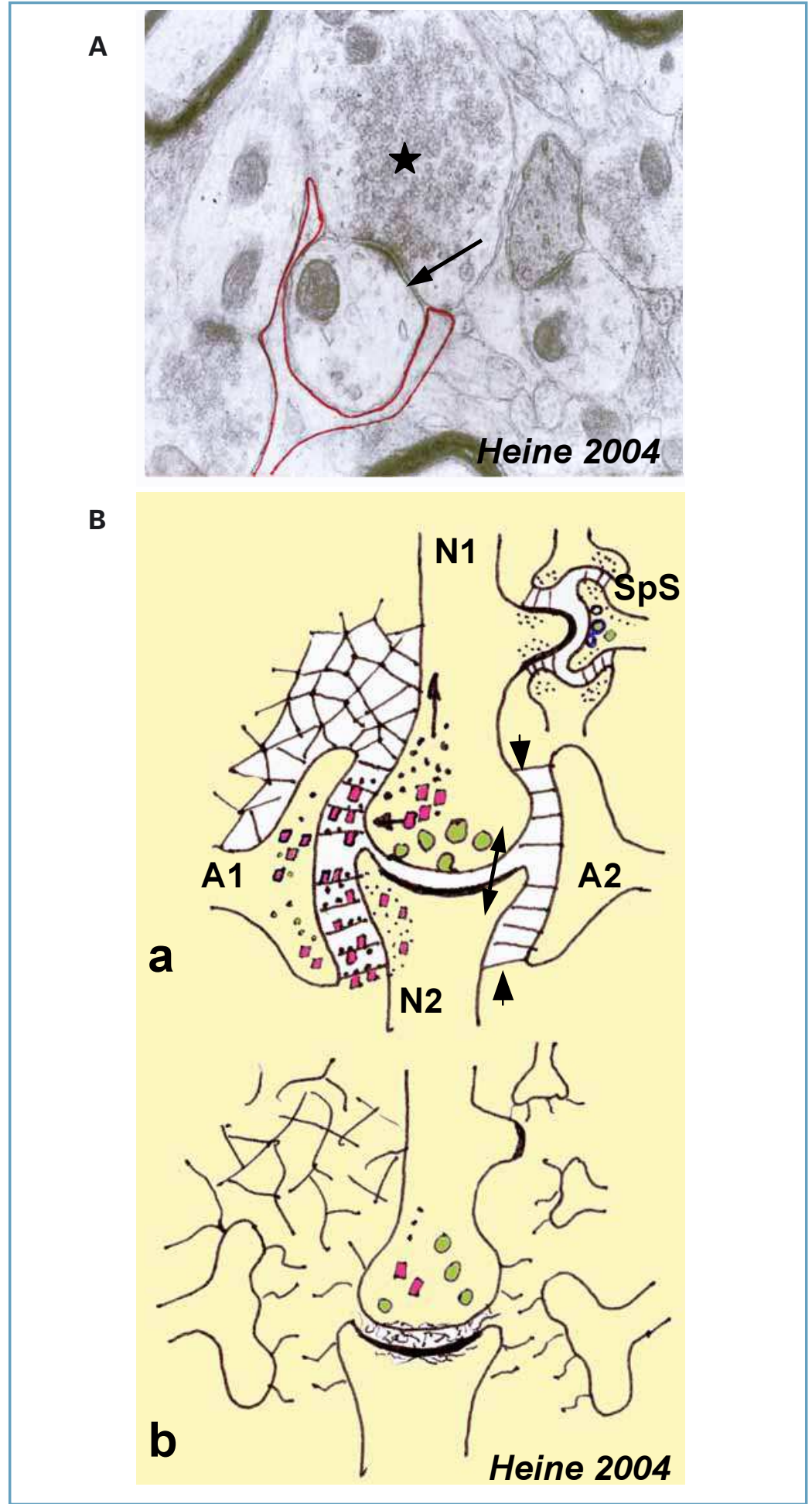

Abb. 5. Schematische Darstellung der Ammenfunktion der Astrozyten an Synapsen. A Gehirn einer Maus. Abschirmung einer Synapse durch Fortsätze eines Astrozyten (durchgehende Linie). Der Pfeil weist auf die Synapse. Der Stern kennzeichnet den präsynaptischen Bereich eines Neuriten gefüllt mit Neurotransmittervesikeln. (7.500-fache Vergr.). B Normale und gestörte Ammenfunktion. a Die Synapse (Doppelpfeil) zwischen dem Nervenzellfortsatz (Dendrit) (N1) und (N2) wird durch verbreiterte Enden von Astrozyten (A1, A2) mit Mikrodomänenbildung (Pfeilköpfe) stabilisiert. Auf der linken Seite ist der Shuttle-Prozess zwischen Astrozyt und Nervenzellfortsatz dargestellt (Punkte). Der nach links gerichtete Pfeil zeigt den Shuttle-Betrieb zwischen Nervenzellfortsatz und Astrozyten (Quadrate). Der Pfeil in Längsrichtung von N1 weist auf den Transport der aufgenommenen Moleküle in Richtung Zelllaib des zugehörigen Neurons. SpS Synapsen an einem Dendritendorn (Spine), abgesichert durch Astrozytenfortsätze mit Mikrodomänen. b Destruierte Mikrodomänen und Synapsen im Bereich einer Alzheimer Plaque. 
rone allmählich vom Nachschub an Cholin abgeschnitten werden. Auf diese Weise werden u. a. die Assoziationsbahnen im Bereich des Hippocampus zunehmend funktionell geschädigt mit Verlust von Lern- und Gedächtnisleistungen [6].

Bei altersbedingter Abnahme der PG/GAGs kann die Netzstruktur der PECM jedoch nur gehalten werden, wenn entweder mehr PG/GAGs gebildet werden, was aufgrund des Enzymmangels nicht möglich ist, oder sich die Halbwertszeiten der PG/GAGs (ca. 2 Wochen) drastisch verlängern. Dies trifft über den Mechanismus der nichtenzymatischen Glykosilierung bei altersbedingten Glukoseverwertungsstörungen zu [22]. Die dabei entstehenden Advanced Glycation Endproducts (AGEs) sind unlösliche komplexe Karbohydratstrukturen [32]. Mikrogliazellen versuchen zwar, derartige AGEs zu phagozytieren, was ihnen anscheinend über lange Zeit auch gelingt. Werden AGEs beschleunigt gebildet (zunehmender Altersdiabetes, Sauerstoffmangel), können sie einen Umfang erreichen, der zu einer „frustranen“ Phagozytose führt, mit Freisetzung von Sauerstoffradikalen (ROS) und lysosomalen Proteasen [32]. Dadurch werden vermehrt Appikanmoleküle in plaquebildende $\mathrm{A} \beta$-Proteine gespalten, für die bereits vorhandene AGEs zu Kondensationspunkten und Katalysatoren der Plaquebildung werden [32]. Deshalb wachsen und vermehren sich die Plaques unter Einschluss von AGEs, Cholesterin und Entzündungsmediatoren [1,32]. Die A $\beta$-Proteine wirken zusätzlich chemotaktisch auf Mikrogliazellen, wodurch die entzündliche Plaquebildung weiter verstärkt wird [32]. Die Mikrodomänen zwischen Astrozyten und neuronalen Synapsen werden im Plaquebereich destruiert, die Synapsenfunktion wird irregulär bzw. eingestellt [22] (Abb. 5).

Im Zuge der extrazellulär entstehenden Plaques kommt es sekundär zu einer typischen Reaktion der betroffenen Neurone: Sie beginnen mit einer Reaktivierung des Zellzyklus, der jedoch nicht mit der Zellteilung abschliesst; zuvor wird aufgrund von Substratmangel das Apoptoseprogramm gestartet [10]. Dies geschieht jedoch erst dann, wenn sich im Verlauf der Mangelversorgung intraneuronal gebildete hyperphosphorilierte Tau-Proteine an das Zytoskelett anlagern und sog. „tangels“ (Fibrillengespinst) bilden. Sie vernetzen das Zytoskelett, stören damit den Zellstoffwechsel und starten dadurch die Apoptose [10,51]. AGEs und die in diese pathologische PECM eingebetteten Neurone behalten durch diese „Teilreaktivierung“ zunächst ihre Grösse. Dadurch wird eine „Pseudotensegrität“ vorgetäuscht, die die, wenn auch geminderte Hirnleistung über eine individuell lange Zeit halten kann. Die vom Autor entwickelte Theorie, dass sich zuerst die Plaque in der PECM bildet und dann erst die TangleBildung mit Neuronenuntergang folgt [22], hat sich unterdessen bestätigt! [5].

Die Plaque zeigt schliesslich eine bestimmte Pathomorphologie (Abb. 6): Um einen amorphen PECM-A $\beta$-Kern liegt ein Hof aus Mikrogliazellen, dem aussen Astrozyten folgen. Die zwischen diesen auftretenden irregulären Dendritensprossungen stellen einen frustranen Versuch zur Wiederherstellung von Synapsen dar [32]. In der Umgebung finden sich apoptotische Neurone mit tangle-Bildung (Abb. 6). Das entzündliche Geschehen bleibt jedoch auf das Gehirn begrenzt und ist erstaunlicherweise ödemfrei, d. h. das adaptive Immunsystem (B- und T-Lymphozyten) wie auch Granulozyten sind nicht beteiligt [15,32]. Wegen der Ödembildung wäre dies mit dem Leben nicht vereinbar. Diese Sonderform der Entzündung dürfte auch Ursache dafür sein, dass die Betroffenen die Erkrankung über viele Jahre bis Jahrzehnte nicht bemerken [15,32]. Dass das adaptive Immunsystem ferngehalten wird, scheint mit der alterungsbedingt starken Verbreiterung und damit Umstrukturierung der Basalmembran der Blut-Hirn Schranke zu tun zu haben $[22,55]$. Dadurch wird deren Astrozyten- und Endothelschranke so verändert, dass sie keine von ihnen produzierte Chemokine und Zytokine mehr passieren lassen kann. Damit Lymphozyten und Granulozyten die Endothelschranke passieren können, müssten auch bestimmte Adhäsionsmoleküle (ICAM-1, VCAM-1, E-Selek- tin) auf dem Kapillarendothel exprimiert werden, die jedoch in den Gehirnkapillaren fehlen [Übersicht bei 24].

\section{Therapeutische Konsequenzen}

Aus biologisch-medizinischer Sicht wäre der beste Weg, zur Verhinderung der Entwicklung einer AD bereits bei Kindern durch Erziehung Strategien zur Stressbewältigung zu vermitteln [6]. Treten jedoch im späteren Leben erste Anzeichen einer AD auf, dann „brennt zwar der Waldrand“, aber die weitere Entwicklung kann therapeutisch verzögert werden. Das Wichtigste ist dabei die Umstellung der Lebensführung (vor allem geistige und körperliche Aktivität) [46]. Das Vorhandensein zahlreicher Zähne im Alter konnte als ein Schutzfaktor vor $\mathrm{AD}$, nicht aber vor einer vaskulären Demenz oder einem ischämischen Insult, erkannt werden [12]. Der Zusammenhang von zuckerreicher Ernährung und Zahnkaries ist wissenschaftlich gesichert. Deshalb sind möglicherweise die noch erhaltenen Zähne der Nichtdementen eine Art „Langzeitgedächtnis“ für eine lebenslange bessere Mundhygiene und/oder gesündere Ernährungsweise, die eventuell vor Alzheimer schützt [12].

Aufgrund der Beziehungen von AD zum Entzündungsgeschehen werden derzeit auch Entzündungshemmer (nichtsteroidale Antiphlogistika, u. a. Azetylsalicylsäure, Ibuprofen und COX-2-Hemmer) geprüft, ohne dass bisher ein Nutzen erkennbar gewesen wäre. Das Gleiche gilt für Statine [2, 10]. Vakzine könnten zur kausalen Behandlung dienen, mussten aber trotz grosser Erfolge im Tiermodell beim Menschen wegen der Gefahr der Entwicklung von Enzephalitis wieder in die vorklinische Untersuchung zurück [5]. Die Übererregung glutamaterger Synapsen bei AD kann durch Glutamat-Antagonisten wie Memantine gedämpft werden, auch Acetylcholinesterasehemmer können eingesetzt werden. Die Erfolge sind jedoch gering und lediglich symptomatisch [2,22]. Geprüft werden auch Östrogene gegen A $\beta$-Bildung [6,10,25]. 


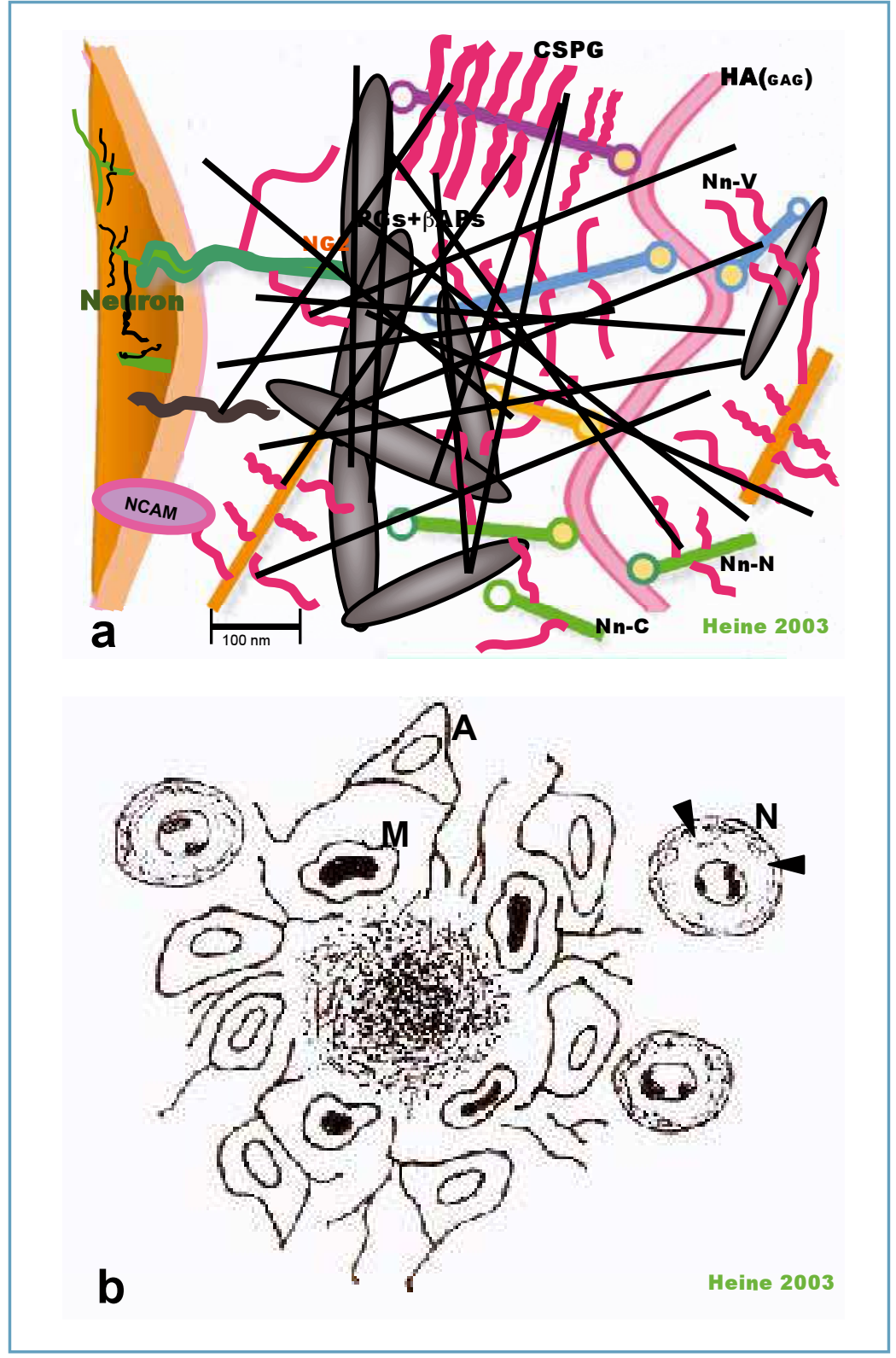

Abb. 6. Plaquebildung in der PECM. a Feinstrukturelles Schema der beginnenden Plaquebildung durch starke Vermehrung und Polymerisation amyloidogener Spaltprodukte unter Einschluss aller PECM Komponenten (PGs + $\beta A P s)$. Das Neuron reagiert darauf mit "tangle" (Gespinnst) Bildung (vom Zytoskelett abzweigende schwarze Fäden). b Lichtmikroskopisches Schema einer Alzheimer Plaque. Um ein amorphes Zentrum aus A $\beta$-Proteinen und destruierten PECM Komponenten liegt ein Hof aus Mikrogliazellen (M), dem nach aussen Astrozyten (A) folgen. Dazwischen irreguläre Astrozytenfortsätze. Peripher liegen im Untergang begriffene Neurone (N) mit „tangle" Bildung (Pfeilköpfe) (ca. 500-fach Vergr.)

Im Laufe des Lebens werden durch AGE- und überschiessende ROS-Bildung nicht nur das Molekularsieb von ECM und PECM in Verschlackungsprozesse getrieben, sondern auch die Bildung wichtiger Enzyme, u. a. des Intermediärstoffwechsels, herabgesetzt. Dabei führt die dramatische Abnahme der Glutaminsynthetase bei
$\mathrm{AD}$ u. a. nicht nur zu einer Abnahme von Glukosamin, dem Grundbaustein der PG/GAGs, sondern auch zu einer erheblichen Zunahme von Ammoniak im Gehirn (evtl. verstärkt durch zusätzlichen Ammoniak bei bestehenden Leberfunktionsstörungen wie der portosystemischen Encephalopathie [21]). Ammoniak wirkt zwar aktivierend auf die zerebrale Glutaminsynthetase, führt aber bei Überschuss zu Schwellung und Funktionsminderung der Astrozyten, so dass der Ammoniakabbau nicht mehr vollständig bewältigt werden kann und neurotoxisch wirkt. Diese die AD beschleunigende Situation ist für die Therapie bisher nicht berücksichtigt worden. Das Ammoniakproblem bei AD sollte intensiver untersucht werden, zumal für die Ammoniakentgiftung gut wirksame Medikamente auf L-Ornithin-L-Aspartat-Basis zur Verfügung stehen [21,54]. Ein Naturpräparat (Matricell ${ }^{\circledR}$ ) aus Bienenprodukten (Weiselsaft der Bienenkönigin (Gelee Royal), Propolis) sowie aufgeschlossenem Blütenpollen, das sich bei Befindensstörungen, wie sie auch bei $\mathrm{AD}$ auftreten (u. a. Vergesslichkeit, Müdigkeit, Depression), bewährt hat [23], wird derzeit auf seine Fähigkeit untersucht, überschüssigen Ammoniak abzufangen. Das Präparat verfügt über einen erheblichen Gehalt an Aminosäuren, wie Arginin und Asparagin, die den Ammoniak verbrauchenden Harnstoffzyklus in den Zellen aktivieren [vgl. 54].

\section{Funding}

Die vorliegende Arbeit wurde finanziell unterstützt durch die Firma St.Johanser Naturprodukte, DE-82119 Gauting.

\section{Literatur}

1. Arlt S, Kontush A, Müller-Thomsen T et al: Lipoproteinoxidation als gemeinsamer Pathomechanismus der koronaren Herzerkrankung und der Alzheimer-Krankheit. Z Gerontol Geriat $2001 ; 34: 461-65$

2. Bachurin SO: Medical chemistry apraches for the treatment and prevention of Alzheimer's disease. Med Res Rev 2003;23:48-88.

3. Barres BA, Smith SJ: Cholesterol-making or breaking synapse. Science 2001;294:12961297

4. Beattie EC, Stellwagen D, Wade M: Control of synaptic strength by glial TNF507 Science 2002:295:2282-2285.

5. Beckmann M: Untangling Alzheimer's by paring plaques bolsters amyloid theory. Science 2004;305:762 (Kommentar zu neuen Arbeiten zur Entstehung und Therapie von Alzheimer-Demenz).

6. Braak H: Neuropathologie bei Morbus Alzheimer. In Zanella F, Kornhuber J (Hrsg.): Fortschritte in der Behandlung des Morbus Alzheimer. Diagnostische und therapeutische Innovationen. Neu-Isenburg: Lingua Med Verlags-GmbH 2003, S. 23-36.

7. Brandt I: Kopfumfang und Gehirnentwicklung. Klin Wochenschr 1981:59:995-1007. 
8. Chiquet M: Regulation of extracellular matrix gene expression by mechanical stress. Matrix Biology 1999;18:417-426.

9. Clara M: Das Nervensystem des Menschen. 2. Aufl. Leipzig: JA Barth 1958, S. 206-209.

10. Copani A, Sortino MA, Nicoletti F, et al Alzheimer's disease research enters a "new cycle": how significant? Neurochem" Res 2002;27:173-174.

11. Cummings JL: Alzheimer's Desease. N Engl J Med 2004;351:56-67.

12. Dienel $M$ : Ein neu entdeckter Schutzfaktor vor Alzheimer-Demenz: Zahlreiche noch erhaltene Zähne - Die Neuburger Demenzstudie. Euro J Ger 2006;8:166-170.

13. Dustin ML, Colman DR: Neural and immunological synaptic relations. Science 2002;298 785-789.

14. Fidler PS, Schuette K, Asher RA, et al: Comparing astrocytic cell lines that are inhibitory or permissive for axon growth. The major axon-inhibitory proteoglycan is NG 2. J Neursci 1999;19:78-88.

15. Finch CE, Cohen DM: Aging metabolism, and Alzheimer's disease: review and hypotheses. Exp Neurol 1997;143:82-102.

16. Fox K, Caterson B: Freeing the brain from the perineuronal net. Science 2002;298:1187-89.

17. Fröhlich E, Hoyer S: Zur ätiologischen und pathogenetischen Heterogenität der Alzheimer Krankheit. Nervenarzt 2002;73:422-427.

18. Füsgen I: Demenz, praktischer Umgang mit Hirnleistungsstörungen. Urban und Vogel: München 2001.

19. Gallo V, Chittajallu R: Unwrapping glial cells from the synapse: What lies inside? Science 2001;292:872-873.

20. Golde TE: Inflammation takes on Alzheimer disease. Nature Medicine 2002;8:936-938.

21. Häberle J, Koch HG: Hyperammonämie: Ursachen, Diagnostik, Therapie. Teil 2: Krankheitsbilder und Therapie. Dtsch Med Wochenschr 2004:129;1430-1433.

22. Heine $\mathrm{H}$ : Die perineuronale Matrix bei Alzheimer Demenz. Teil 1 u. 2. Geriatrie Journa 2004:Heft 1:31-36: Heft 2:41-45

23. Heine $H$, Andrä F: Anregung der immunologischen Beistandsreaktion mit Bienenprodukten und aufgeschlossenem Blütenpollen. Erfahrungsheilkunde 2006;55:1-6.

26. Hogan I: Komplexität in der Krise. Spektrum der Wissenschaft 1995; Heft 9: 58-64.

27. Ikegaya $Y$, Aaron G, Lossart R, et al: Synfire chains and cortical songs: temporal modules of cortical activity. Science 2004;304:559564.

28. Ingber DE: Architektur des Lebens. Spektrum der Wissenschaft 1998; Heft 3:32-40.

29. Iozzo RV: Matrix proteoglycans: from molecular design to cellular function. Annu Rev Biochem 1998;67:609-652.

30. Kasischke KA, Vishwasrao HD, Fischer PJ et al: neural activity triggers neuronal oxidative metabolism followed by astzrocytic glycolysis. Science 2004;305:99-103.

31. Kindzelskii AL, Petty HR: Apparent role of traveling metabolic waves in periodic oxidant release by living cells. Proc Nat Acad Sci USA 2002;99:9207-9212.

32. McGeer PL, McGeer EG: The inflammatory response system of brain: implications for therapy of Alzheimer and other neurodegen erative disease. Brain Res Rev 1995;21: 195-218.

33. Marambaud $P$, Wen $P H$, Dutt A: A CBP bind ing transcriptional repressor produced by the PS1/epsilon-cleavage of $\mathrm{N}$-cadherin is inhibited by PS1 FAD mutations. Cell 2003:114 635-645.

34. Margolis RK, Margolis R: Nervous tissue proteoglycans. In Jolles P (ed): Proteoglycans Basel-Boston-Berlin: Birkhäuser Verlag 1994 S. 144-177

35. Maurer K: Dem Gehirn beim Denken zuschauen. Zukunftsforum Demenz. Dokumentationsband 3. Medical Tribune Verlagsgesellschaft: Wiesbaden 2003, S. 11-21.

36. Milner R, Campbell IL: The integrin family of cell adhesion molecules has multiple functions within the CNS. J Neurosci Res 2002; 69:2286-2291.

37. Nikolaus S, Schreiber S: Molekulare Mechanismen für die Kontrolle der Lebenserwartung. Dtsch Med Wochenschr 2004;129: 903-907.

38. Nussbaum R, Ellis CE: Alzheimer's disease and Parkinson's disease. N Engl J Med 2003;348:1356-1375

39. Pangalos MN, Efthimiopoulos S, Shioi J et al: The chondroitin sulfate attachment site of appican is formed splicing out exon 15 of the amyloid precursor gene. J Biol Chem 1995, 270:10388-10391.

40. Perumpanani AJ, Simmons DL, Gearing AJ, et al.: Extracellular matrix mediated chemo taxis can impede cell migration. Proc R Soc Lond B 1998;2347-2352.

41. Rees J: Complex disease and the new clinica sciences. Science 2002;296:698-701.

42. Saitoh T, Mook-Jung I: Biological function of APP and Alzheimer's disease. Alzheimer's Dieease Review 1996;1:30-36.

43. Schlesinger J: Autoinhibition control. Scienc 2003;300:750-752

44. Small DH, Mok SS, Williamson TG et al. Role of proteoglycans in neural development, regeneration, and the aging brain. J Neurochem 1996;67:889.

45. Snow DM, Mullins N, Hynds DL: Nervous system-derived chondroitin sulfate proteoglycans regulate growth cone morphology and inhibit neurite outgrowth: a light, epifluorescence, and electron microscopy study. Microsc Res Tech 2001:54:273-286.

46. Solfrizzi V, Panza F, Capurso A: The role of diet in cognitive decline. J Neural Transm 2003;110:95-110.

47. Streuli C: Extracellular matrix remodelling and cellular differentiation. Curr Opin Cell Bio 1999;11:634-640.

48. Strohmann R: Epigenesis: The missing beat in biotechnology. Biotechnology (N Y) 1994; 12:156-164.
49. Stuhlsatz HW, Greiling H: Proteoglycans and glycosaminoglycans of human joint cartilage in health, senescence, and disease. In Varma RS, Varma R (eds): Glycosaminoglycans and Proteoglycans in Physiological and Pathological Processes of Body Systems. Basel: Karger 1982, S. 276-289.

50. Tadokoro S, Shattil S, Eto K, et al: Talin binding to integrin tails. A final common step in integrin activation. Science 2003;302:103-106.

51. Taylor JP, Hardy J, Fischbeck KH: Toxic proteins in neurodegenerative disease. Science 2002;296:1991-95

52. Teipel St: Morbus Alzheimer: 100 Jahre "Krankheit des Vergessens". Dtsch Med Wochenschr 2006;131:1991-1992.

53. Torre JC de la: Critical threshold cerebral hypoperfusion causes Alzheimer's disease. Acta Neuropathol 1999;98:1-8.

54. Willems R-E, Heine $\mathrm{H}$ : Gibt es einen gemeinsamen pathophysiologischen Hintergrund der Altersdemenzen? Arztezeitschr $f$ Naturheilverf 2004;45:12;800-801.

55. Xi YP, Nette D, King W, Rosen M: Accerelated changes in normal human basement membrane. Mech Age Develop 1982;19: 315-324

56. Yosojima K, McGeer EG, McGeer PL: Relationship between amyloid peptide generating molecules and neprilysin in Alzheimer's disease and normal brain. Brain Res 2001;919: 115-121.

57. Zilles K, Rehkämper G: Funktionelle Neuroanatomie. 3. überarb. Aufl Berlin HeidelbergNew York: Springer 1998.

\section{Korrespondenzadresse}

Prof. Dr. rer. nat. med. habil. Hartmut Heine Billerbeckweg 1-3, DE-75242 Neuhausen hartmutheine@aol.com 